\title{
Circuit
}

Musiques contemporaines

\section{In memoriam Christian Bourgois (1933-2007)}

\section{Pierre Boulez et Jean-Jacques Nattiez}

Volume 19, numéro 2, 2009

Stockhausen au Québec

URI : https://id.erudit.org/iderudit/037467ar

DOI : https://doi.org/10.7202/037467ar

Aller au sommaire du numéro

Éditeur(s)

Les Presses de l'Université de Montréal

ISSN

1183-1693 (imprimé)

1488-9692 (numérique)

Découvrir la revue

Citer ce compte rendu

Boulez, P. \& Nattiez, J.-J. (2009). Compte rendu de [In memoriam Christian Bourgois (1933-2007)]. Circuit, 19(2), 99-101. https://doi.org/10.7202/037467ar d'utilisation que vous pouvez consulter en ligne.

https://apropos.erudit.org/fr/usagers/politique-dutilisation/ 


\section{ACTUALITÉS}

\section{In memoriam Christian Bourgois (1933-2007)}

Pierre Boulez et Jean-Jacques Nattiez

À la suite de la disparition du grand éditeur français, ardent défenseur du livre sur la musique contemporaine, Circuit publie deux hommages, signés Pierre Boulez et Jean-Jacques Nattiez, deux auteurs ayant été publiés chez Christian Bourgois Éditeur.

Publier une collection de livres sur la musique, plus spécifiquement sur la musique du $\mathrm{xx}^{\mathrm{e}}$ siècle, quelle utopie, et même quelle folie irresponsable! De cette folie, de cette utopie, Christian Bourgois avait décidé d'assumer la responsabilité pleine et entière, en parfaite connaissance de cause. On lui doit non seulement un fort sentiment d'obligation, mais, bien plus, une gratitude reconnaissante pour avoir compris que la musique, composante capitale de la vie intellectuelle, mérite que l'on s'y intéresse certes par l'exécution d'œuvres encore trop rarement inscrites dans les programmes courants ou d'œuvres absolument nouvelles, mais tout autant par l'étude de leurs origines, des courants de pensée qui leur donnent, ou leur ont donné, naissance, voire de la technique elle-même par laquelle elles se transmettent. Malheureusement, l'éducation artistique trop chichement répandue entretient le lieu commun que la musique n'a aucunement besoin de ces béquilles savantes, l'intuition et l'émotion suffisant à une appréciation absolue, au-delà de toute contingence analytique, stigmatisée comme une erreur fondamentale sur la catégorie et la réalité de la perception. Ainsi, qu'un éditeur ne se soit pas résolu à la pauvreté, à l'indigence de ce type de réactions, mais qu'il ait persévéré au-delà de toutes les difficultés qu'un tel projet suscite inévitablement, mérite qu'on considère l'exemple de Christian Bourgois à l'échelle de son courage, de sa perspicacité et de sa lucidité.

Pierre Boulez

Baden-Baden, mars 2008 
Le 20 décembre 2007 disparaissait, des suites d'une douloureuse maladie, un des éditeurs qui, de par son audace, aura le plus compté dans la vie intellectuelle contemporaine de la francophonie. Fondateur de la célèbre collection de poche 10-18 et de la maison d'édition qui porte son nom, il n'aura cessé de publier tout ce que la pensée française a pu proposer d'avantgardiste à l'époque de la modernité. Éditeur, dans le domaine littéraire, aussi bien des romans de Boris Vian que du théâtre d'Arrabal, des Versets sataniques de Salman Rushdie - à ses risques et périls - que de Jorge Luis Borges, il accordait une attention particulière à la littérature anglophone contemporaine (Edward Bond, William Burroughs, Leonard Cohen, Toni Morrison, Gertrude Stein, Virginia Woolf parmi bien d'autres). Mais pourquoi une revue qui se consacre à la musique contemporaine lui rend-elle un hommage? Parce que notre communauté lui doit beaucoup.

En 1975, il acceptait, sur la recommandation de Mikel Dufrenne et de Daniel Charles, de publier en 10-18 le premier livre de l'auteur de ces lignes. Dans la foulée de cette première collaboration, il accueillit la collection que Pierre Boulez m'avait proposé de codiriger avec lui, Musique/passé/présent. Il convient de souligner l'audace, voire la témérité, de Christian Bourgois, alors que trois éditeurs parisiens, et non des moindres, avaient refusé ce projet ou vite déclaré forfait. Lorsque je le lui proposai, il n’hésita pas une seule seconde: sa vaste culture et sa perspicacité aiguë lui dictaient qu'il fallait publier les textes des compositeurs contemporains, qu'il y avait là une nécessité intellectuelle, quelles que soient les difficultés matérielles de l'entreprise. C'est ainsi que paraîtront en 1981 et 1985 les deux éditions des Points de repère de Pierre Boulez $(1981,1985)$ et, en 1989, une première mouture de ses cours du Collège de France - Jalons (pour une décennie) - puis sa correspondance avec John Cage (1991), un dialogue sur la direction d'orchestre, L'écriture du geste (2002), puis la réédition complète de ses écrits selon des principes différents: Points de repère, I - Imaginer (1995), Regards sur autrui (Points de repère, II) (2005), Leçons de musique (Points de repère, III) qui couvrent la totalité de l'enseignement de Pierre Boulez au Collège de France (2005). Il accueillit aussi les écrits de Steve Reich (1981), Mauricio Kagel (1983), Edgar Varèse (1983), Alban Berg (1985), Hugues Dufourt (1991) et Luigi Nono (1993). Également plusieurs ouvrages de musicologie, de musicographie et d'esthétique musicale: outre les travaux de l'auteur de ces lignes, Wozzeck d'Alban Berg de Pierre Jean Jouve et Michel Fano (1985), Quoi? quand? comment? la recherche musicale, un colloque de l'IRCAM (1985), Invention musicale et idéologies de Célestin Deliège (1986), Le cerveau et la musique 
de Jean-Paul Despins (1986), Du beau dans la musique d’Édouard Hanslick (1986), Le Don Juan de Mozart de Pierre Jean Jouve (1986), le Edgard Varèse de Fernand Ouellette (1989), Qu'est-ce que la musique? de Roman Ingarden (1989), Le timbre, métaphore pour la composition, un recueil préparé par Jean-Baptiste Barrière (1991), La classe de Messiaen de Jean Boivin (1995). Du côté des interprètes, il faut encore citer la correspondance de Glenn Gould (1992), sa Série Schönberg (1998), les poèmes d'Alfred Brendel (2001) et Le voile de l'ordre (2002), un long entretien avec le pianiste, sans oublier l'imposante discographie de la Callas commentée par Réal La Rochelle (1997).

On aura reconnu au passage le nom de quelques auteurs québécois (Boivin, Despins, Ouellette, La Rochelle) auxquels il convient d'ajouter ceux de traducteurs, «éditeurs » ou préfaciers d'ici : Annick Duchâtel, Jonathan Goldman, Ghyslaine Guertin, Caroline Guindon, Louise Hirbour, Christiane Léaud, Gaétan Martel, Stéphane Roy, Dujka Smoje.

Avec la disparition de Christian Bourgois, le milieu musical francophone, et tout particulièrement celui qui s'intéresse de près à la musique du $\mathrm{Xx}^{\mathrm{e}}$ siècle, a perdu un éditeur militant et convaincu sans lequel notre connaissance de la culture d'aujourd'hui n'aurait pas été ce qu'elle est.

Jean-Jacques Nattiez

Montréal, janvier 2009 